\title{
Drug transport and metabolism in the blood-brain barrier
}

\author{
Jaime Kapitulnik* \\ Department of Pharmacology, Institute of Drug Research, School of Pharmacy, Faculty of Medicine, The Hebrew University of Jerusalem, Jerusalem, Israel \\ ${ }^{*}$ Correspondence: jaimek@savion.huji.ac.il
}

Despite recent advances, the treatment of brain diseases and neurological disorders, such as the Parkinson and Alzheimer syndromes, multiple sclerosis, brain cancer, psychiatric disorders, many forms of epilepsy, chronic pain, and migraine, remains a major challenge. There is a growing need for the development of effective CNS-active drugs that are able to reach the desired regions of the brain. This has proven to be problematical, as the brain is protected very effectively against exogenous substances, including many potentially useful CNS-active drugs. This issue has been discussed in detail at a EU-COST Exploratory Workshop which produced a policy paper for the EU (Kapitulnik et al., 2010).

Access to brain tissue and ensuing effects of drugs or other chemicals are dependent on their ability to cross the blood-brain barrier (BBB; Pelkonen et al., 2008; Kapitulnik et al., 2009). The BBB is a physical and functional barrier that prevents the entrance of many drugs and chemicals into brain cells regardless of their molecular size. The BBB controls access to the brain of essential nutrients, vitamins and ions, as well as some proteins and peptides, and removes the products of metabolism in the brain, for example neurotransmitter metabolites. It also prevents entry of potentially harmful substances into the brain (e.g., bilirubin, an endogenously formed neurotoxic catabolite of heme, as well as exogenous toxins).

The BBB comprises the endothelial cells of small blood vessels. The fact that endothelial cells of brain capillaries differ greatly from those in the periphery confers on the $\mathrm{BBB}$ its discriminatory characteristics. Brain endothelial cells display tight junctions, absence of intercellular clefts and fenestrations, minor pinocytic activity, and a high electrical resistance. These cells have a continuous basal membrane and are surrounded by pericytes and the end feet of astrocytes, which are part of the $\mathrm{BBB}$ and control its permeability (reviewed by Abbott et al., 2010).
Proteins involved in drug transport (drug transporters, DTs) and metabolism (drug metabolizing enzymes, DMEs) have been described in many organs (liver, lung, kidney, intestine, brain, skin, blood vessels, and others). These proteins play important roles by governing both the traffic between organs and elimination of drugs and foreign compounds (xenobiotics), as well as in both synthesis and degradation/elimination of endogenous compounds (endobiotics) such as hormones and prostanoids. In certain instances, their action is beneficial (detoxification of both xenobiotics and endobiotics), but they may also display deleterious effects, as is the case with certain DTs which do not allow drugs to enter the brain or DMEs that activate xenobiotics (e.g., drugs and chemical carcinogens) to form toxic metabolites (for detailed reviews see Dutheil et al., 2010; Miller, 2010). Some of the genes that regulate the expression of DTs and DMEs have been shown to be polymorphic, resulting in the synthesis of proteins with impaired or increased activities (Ieiri et al., 2009; Cascorbi and Haenisch, 2010; Franke et al., 2010; Johansson and Ingelman-Sundberg, 2011). These polymorphisms may thus profoundly affect the blood levels of drugs and chemical toxins.

Drug transporters and DMEs are also present in the BBB. Using transcriptomics and proteomics approaches, the presence of these proteins has been demonstrated in isolated human brain microvessels and cortex biopsies (Shawahna et al., 2011). DTs and DMEs control the access to the brain and local concentration of both endobiotics and xenobiotics. Transport proteins enable passage of those substances required by the CNS, such as glucose, essential amino acids, and neurotransmitter precursors. There are also transporters and metabolic enzymes that function in the opposite direction, thereby preventing access to the brain of some lipid-soluble drugs and potentially toxic substances, including metabolites, which might otherwise be able to diffuse into the brain and cause damage. Unfortunately, this means that the BBB can also prevent otherwise effective drugs from entering the brain. It may also hinder side effects of these and other drugs. The number of these drugs is larger than once thought.

Moreover, the function of the DTs at the $\mathrm{BBB}$ is also affected during disease (Zlokovic, 2008; Engelhardt and Sorokin, 2009), resulting in enhanced exposure of the CNS to neurotoxic compounds and reduced drainage of CNS-born proteins such as amyloid proteins, thus promoting neurodegeneration. Today we still know very little about how inflammatory components of systemic or CNS diseases influence drug transport into the brain, and how peptides and proteins are taken across the BBB.

The importance of active transporters and local metabolism in the BBB in causing harmful effects of drugs and environmental chemicals remains largely unknown. Transporters and enzymes are known to work in conjunction with each other (e.g., in the intestine and liver; Benet, 2009), but it is not known how they are coordinated at the $\mathrm{BBB}$, or how important enzymes are relative to transporters at the $\mathrm{BBB}$. Thus it is of utmost importance to characterize the expression, activity and physiological and pathological regulation of these proteins in the BBB and adjacent brain areas, both in vivo and in model systems.

The methods currently used to study the transport and metabolism of drugs and toxins in the BBB and adjacent brain areas include a battery of probes for measuring the expression of transporter and enzyme genes in appropriate in vitro and in vivo model systems, a battery of substrate probes for various transporters and xenobiotic-metabolizing enzymes to be studied in the model systems, immunochemical techniques for studying the localization and architecture of transporters and enzymes in brain structures, and imaging techniques to investigate timeand site-dependent processes of transport 
and metabolism. Basic characterization of all developed model systems with the help of the above mentioned methods is a necessary prerequisite for the extensive application of the models for other types of studies. Moreover, development of new models and methods is also of prime importance.

\section{FUTURE CHALLENGES}

1. Although important knowledge on DTs in blood-brain and blood-CSF interfaces has accumulated during the last decade, it is still very limited. However, it is becoming clear that the battery of DTs expressed in these interfaces is somewhat different than in peripheral organs, thus making it necessary to carefully identify the DTs in the above interfaces as well as understanding their regulation and function.

2. The available knowledge on DMEs in these interfaces is rather limited, and it is thus imperative to further study which are the enzymes expressed in these tissues.

3. Given the known interplay between DTs and DMEs in liver and intestine, it is important to determine if these proteins work in tandem in the CNS or compete for their substrates.

The results of studies on the interactions between enzymes and transporters, and between drugs and endogenous compounds, as well as on the role of enzyme and transporter polymorphisms in determining brain access and active site concentration of drugs, will contribute to the therapeutic impact of existing and newly designed CNS drugs, as well as to the prevention of the toxic effects of xenobiotics.

\section{REFERENCES}

Abbott, N. J., Patabendige, A. A. K., Dolman, D. E. M., Yusof, S. R., and Begley, D. J. (2010). Structure and function of the blood-brain barrier. Neurobiol. Dis. 37, 13-25.

Benet, L. Z. (2009). The drug transporter - metabolism alliance: uncovering and defining the interplay. Mol. Pharmacol. 6, 1631-1643.

Cascorbi, I., and Haenisch, S. (2010). Pharmacogenetics of ATP-binding cassette transporters and clinical implications. Methods Mol. Biol. 596, 95-121.

Dutheil, F., Jacob, A., Dauchy, S., Beaune, P., Scherrmann, J.-M., Decleves, X., and Loriot, M.-A. (2010). ABC transporters and cytochromes $\mathrm{P} 450$ in the human central nervous system: influence on brain pharmacokinetics and contribution to neurodegenerative disorders. Expert Opin. Drug Metab. Toxicol. 6, 1161-1174.

Engelhardt, B., and Sorokin, L. (2009). The bloodbrain and the blood-cerebrospinal fluid barriers: function and dysfunction. Semin. Immunopathol. 31, 497-511.

Franke, R. M., Gardner, E. R., and Sparreboom, A. (2010). Pharmacogenetics of drug transporters. Curr. Pharmacol. Design 16, 220-230.

Ieiri, I., Higuchi, S., and Sugiyama, Y. (2009). Genetic polymorphisms of uptake (OATP1B1,1B3) and efflux (MRP2, BCRP) transporters: implications for interindividual differences in the pharmacokinetics and pharmacodynamics of statins and other clinically relevant drugs. Expert Opin. Drug Metab. Toxicol. 5, 703-729.

Johansson, I., and Ingelman-Sundberg, M. (2011). Genetic polymorphism and toxicology - with emphasis on cytochrome P450. Toxicol. Sci. 120, 1-13.
Kapitulnik, J., Hammarlund-Udenaes, M., GundertRemy, U., Kostelidou, K., Crichton, R., Zarkovic, N., and Boobis, A. R. (2010). COST Exploratory Workshop: pharmacology and toxicology of the blood-brain barrier: state of the art, needs for future research and expected benefits for the EU. Front. Neurosci. doi: 10.3389/conf.fphar.2010.02.00003

Kapitulnik, J., Pelkonen, O., Gundert-Remy, U., Dahl, S. G., and Boobis,A.R. (2009). Effects of pharmaceuticals and other active chemicals at biological targets: mechanisms, interactions, and integration into PB-PK/PD models. Expert Opin. Ther. Targets 13, 867-887.

Miller, D. S. (2010). Regulation of P-glycoprotein and other ABC drug transporters at the blood-brain barrier. Trends Pharmacol. Sci. 31, 246-254.

Pelkonen, O., Kapitulnik, J., Gundert-Remy, U., Boobis, A. R., and Stockis, A. (2008). Local kinetics and dynamics of xenobiotics. Crit. Rev. Toxicol. 38, 697-720.

Shawahna, R., Uchida, Y., Decleves, X., Ohtsuki, S., Yousif, S., Dauchy, S., Jacob, A., Chassoux, F., Daumas, C., Couraud, P.-O., Terasaki, T., and Scherrmann, J.-M. (2011). Transcriptomic and quantitative proteomic analysis of transporters and drug metabolizing enzymes in freshly isolated human brain microvessels. Mol. Pharmacol. doi: 10.1021/mp200129p. [Epub ahead of print].

Zlokovic, B. V. (2008). The blood-brain barrier in health and chronic neurodegenerative disorders. Neuron 57 , 178-201.

Received: 28 June 2011; accepted: 05 July 2011; published online: 15 July 2011.

Citation: KapitulnikJ (2011) Drug transport and metabolism in the blood-brain barrier. Front. Pharmacol. 2:37. doi: 10.3389/fphar.2011.00037

This article was submitted to Frontiers in Drug Metabolism and Transport, a specialty of Frontiers in Pharmacology. Copyright $(2011$ Kapitulnik. This is an open-access article subject to a non-exclusive license between the authors and Frontiers Media SA, which permits use, distribution and reproduction in other forums, provided the original authors and source are credited and other Frontiers conditions are complied with. 